\title{
Contents/Contenido
}

Acknowledgments/Agradecimientos xi

Abbreviations/Abreviaturas xii

Regarding the Indigenous Languages and Alphabets in This Volume xiii

Acerca de los idiomas y alfabetos indígenas de este volumen xiii

\section{Carlos Montemayor}

Poetry in Mexican Indigenous Languages 1

La poesía en lenguas indígenas de México 11

\section{Donald Frischmann}

Spirit-Matter-Word: Contemporary Mexican Indigenous Poetry 20

Espíritu-Materia-Palabra: Poesía indígena contemporánea de México 31

1. Víctor de la Cruz (Isthmus Zapotec/zapoteco del Istmo) 42 Chupa si diidxa' 44 Just Two Words 45

Sólo dos palabras 45

Dxi biaba' 46

My Fall 47

Mi caída 47

Diidxa' bisiaanda' 46

The Word I Have Forgotten 47

La palabra que olvidé $\quad 47$

Lidxe' canayubi lii 48

My House Seeks You 49

Mi casa te busca 49

Huazacalú 50

It Usually Happens 51

Suele suceder 51

Tu laanu, tu lanu 52

Who Are We? What Is Our Name? 53

¿Quiénes somos, cuál es nuestro nombre? 53

Dxi guyubu' naa 54

When You Search with Your Eyes 55

Cuando con tus ojos busques 55

\section{Víctor Terán}

(Isthmus Zapotec/zapoteco del Istmo) 56

Huadxí que ziyaba 58

The Day Was Fading Away 59

Caía la tarde 59

Ndaani' batanaya' 60

From the Palm of My Hand 61

En la palma de mi mano 61

Lu ti nagana 62

Indecision 63

Duda 63

Xhoopa' diidxa' ruí' xiinga guendaranaxhii 64

Six Variations on Love 65

Seis variaciones acerca del amor 65

Yaga yaa 66

Unseasoned Wood 67

Leña verde 67

Biluxe 68

It's Over 69

Se acabó 69

3. Natalia Toledo Paz

(Isthmus Zapotec/zapoteco del Istmo) 70

Liidxi na Olga 72

Olga's House 73

La casa de Olga 73

Zenaida 74

Zenaida 75

Zenaida 75

Na Marcelina 76

Doña Marcelina 77

Doña Marcelina 77

Yu'du' 78

Temple 79

Templo 79

Yoo lidxe' 80

First House $\quad 81$

Casa primera 81

Guiee lú dani 82

Eye of the Volcano 83

Ojo de volcán 83 


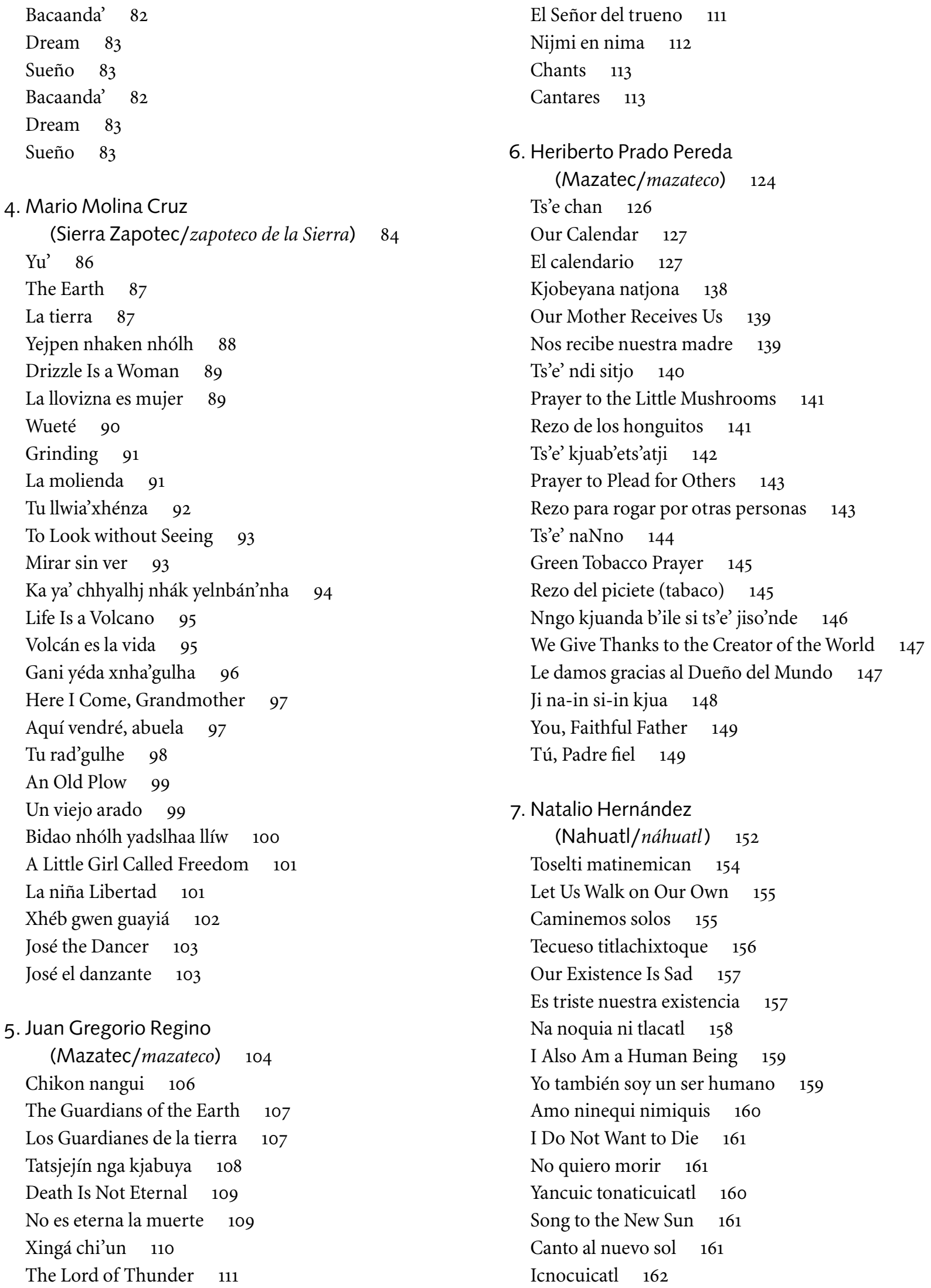


Song of Orphanhood 163

Canto de orfandad 163

Teotlacxochitl 162

Flower of Dusk 163

Flor del atardecer $\quad 163$

Nehuatl nimoyoltlatlanilia 164

I Ask Myself 165

Yo me pregunto $\quad 165$

Itlamiya xochicoscatl 164

Necklace of Flowers 165

Collar de flores $\quad 165$

\section{Serafín Thaayrohyadi Bermúdez}

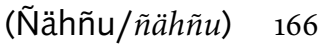

Nzöya xui 168

Sir Night 169

Señor noche 169

Tsi ngande zänä 168

Grandma Moon 169

Abuelita Luna 169

Hin gi 'bụhsehu 170

You Are Not Alone $\quad 171$

No están solos 171

Hiti thähi 172

Spinner 173

Hilandera 173

Dro ñätho ñähñu 'ñuhu 'ñuhmu 172

I Am Ñähñu 173

Soy otomí 173

Tihta 176

Temascal 177

Temascal 177

Tsi Mähkitaa Lerma $\quad 178$

Sacred Father Lerma 179

Sagrado Padre Lerma 179

\section{Briceida Cuevas Cob}

(Mayan/maya) 182

A na' 184

Your Mother 185

Tu madre 185

U ak'abil tu chibil uj $\quad 186$

Night of Eclipse $\quad 187$

Noche de eclipse $\quad 187$

Je bix chuuk 188

Like Charcoal $\quad 189$

Como el carbón $\quad 189$
A yaax tup 188

Your First Earring $\quad 189$

Tu primer arete $\quad 189$

Yan a bin xook 190

You Will Go to School 191

Irás a la escuela 191

Jeex uulich 194

Like a Snail 195

Como caracol de tierra 195

\section{Gerardo Can Pat}

(Mayan/maya) 198

Kin wilik tin wenel 200

I See Her in My Dreams 201

La veo en mis sueños 201

Teech u dzook in tuukul 202

My Final Thought 203

Mi último pensamiento 203

Tii u saas ak'abil abril $\quad 204$

On a Clear April Night $\quad 205$

En una noche clara de abril 205

Teech yeten teen 206

You and I 207

Tú y yo 207

Taan a bin $\quad 208$

You Are Leaving 209

Te vas 209

Xi'iktech utzil 210

I Wish You Well 211

Que te vaya bien 211

K'a'ajsaj 212

Memories 213

Recuerdos 213

\section{Alberto Gómez Pérez}

(Tzotzil/tzotzil) 214

Chavuk 216

Thunder 217

Trueno 217

Kakavute' 218

Cacaté 219

Cacaté 219

K'epelal 220

Drought 221

Sequía 221

Yalemo' 222

The Waterfall 223 
La cascada 223

K'elo me le ixmaltike 224

Watch Over the Corn Fields 225

Cuida los maizales 225

Ja' le kalab kuntikile 226

My Children and Grandchildren $\quad 227$

Mis hijos y nietos 227

Yibelun k'op 228

Source of the Word 229

Raíz de la palabra 229

Mu jna' jayib k'ak'al $\quad 228$

Unaware of the Days 229

Ignoro los días 229

K'unk'un ta k'unk'un 230

Little by Little 231

Poco a poco 231

Muk'ul o'ntonal 232

Consolation 233

Consolación 233

Alboto 232

Tell Him 233

Dile 233

Ilikik 234

They Awakened 235

Despertaron 235

12. Jun Tiburcio

$$
\text { (Totonac/totonaco) } 236
$$

Jununu 238

Jununu 239

Jununu 239

Tachuwin xa tutunaku 240

Sweet Tutunaku Language $\quad 241$

Dulce idioma tutunaku 241

Xtantun xakgalh 242
The Talons of the Eagle 243

Garras de águila 243

Li tutunaku 244

Totonaco! Totonaco! 245

¡Totonaco, totonaco! 245

Mariano Peres Arenal 246

Mariano Pérez Arenal 247

Mariano Pérez Arenal 247

Kaman xalak Chumatlán 248

Chumatec Children $\quad 249$

Niños chumatecos 249

Taskulanatlon 250

Blessings 251

Bendiciones 251

Jun 252

Hummingbird 253

Colibrí 253

Appendices/Apéndices 255

Appendix A. The Owners, Lords, or Guardians of the Earth and the Water 255

Apéndice A. Los Dueños, Señores o Guardianes de la tierra y el agua $\quad 255$

Appendix B. Hurricane and Lightning Entities 257 Apéndice B. Entidades del huracán y del rayo 257

Appendix C. Traditional Prayers and Healing Ceremonies 261

Apéndice C. Rezos tradicionales y ceremonias de curación 261

English Glossary $\quad 265$

Glosario español $\quad 271$

Sources of the Poems/Fuentes de los poemas $\quad 277$ 